DOI 10.37882/2500-3682.2021.07.08

\title{
ВРЕМЯ СУБСТАНЦИАЛЬНОЙ КОНЦЕПЦИИ: ИСТОРИЯ И СОВРЕМЕННОСТЬ
}

\section{TIME OF SUBSTANCIAL CONCEPT: HISTORY AND MODERNITY}

\section{T. Lolaev \\ A. Gabaraev}

Summary: The work is devoted to the history of the substantial concept of time, to the time when it originated and developed, and how I. Newton introduced it into physics with all the ensuing consequences.

In this regard, the article examines the positive and negative aspects of the substantial concept of time, makes generalizations and conclusions about its significance for science in the past and present.

It also emphasizes that A. Einstein rejected the classical concepts of absolute, independent time. Thus, A. Einstein, instead of the substantial concept of time by I. Newton, proposed the relational concept of time, in which time was no longer a physical entity.

In addition, the study reveals the degree of correspondence and inconsistency of the scientific provisions of the substantial and functional concepts of time, developed in the 70s of the last century, in which time, as in the theory of relativity, is nonsubstantial, is not a physical entity. As a result of the analysis of the history of the emergence and evolution of the substantial and other concepts of time, their relationship and confrontation in the light of the current level of their development, it is allowed to create a single concept of time, based on the synthesis of various concepts of time.

Keywords: substantial concept of time, absolute time, relative time, functional concept of time, objectively real time, functional time, nonsubstantial time, eternity of the world, eternity of time.
Лолаев Тотраз Петрович

Д.ф.н., профессор, Северо-Кавказский горно-металлургический институт (государственный технологический университет) lolaev.tp@gmail.com

Габараев Анатолий Дмитриевич

К.ф.н., доцент, Юго-Осетинский государственный университет

gabaraev689@gmail.com

Аннотация: Работа посвящается истории субстанциальной концепции времени, тому, когда она зародилась и развивалась, и как И. Ньютон ввел ее в физику со всеми вытекающими отсюда последствиями.

В этой связи в статье рассматриваются положительные и отрицательные стороны субстанциальной концепции времени, делаются обобщения и выводы о ее значении для науки в прошлом и настоящем.

В ней также подчеркивается, что А. Эйнштейн, отказался от классических представлений об абсолютном, ни от чего не зависящем времени. Тем самым, А. Эйнштейн вместо субстанциальной концепции времени И. Ньютона, предложил реляционную концепцию времени, в которой время уже не являлось физической сущностью.

Кроме того, в исследовании выявляется степень соответствия и несоответствия научных положений субстанциальной и функциональной концепций времени, разработанной в 70-е годы прошлого столетия, в которой время, как и в теории относительности, несубстанционально, не является физической сущностью.

В результате анализа истории возникновения и эволюции субстанциальной и других концепций времени, их взаимоотношения и конфронтации в свете современного уровня их развития допускается создание единой концепции времени, на основе синтеза различных концепций времени.

Ключевые слова: субстанциальная концепция времени, абсолютное время, относительное время, функциональная концепция времени, объективнореальное время, функциональное время, несубстанциональное время, вечность мира, вечность времени.

нятию времени приданы здесь две примечательные функции. С одной стороны, время обусловливает движение атомов (или, что то же самое, вечность времени обусловливает вечность движения); с другой стороны, неограниченность времени обусловливает вечность и неизменность субстанции. Здесь довольно отчетливо проступает двойственная природа времени как условия одновременно и всеобщей изменчивости. и всеобщей сохраняемости (хотя, по всей видимости, ставили акцент на последней). Таким образом, в учении античных атомистов трактовка времени как субстанции не ограничивается утверждением того, что порядок объектов во времени носит самодовлеющий характер. Здесь выдвигается более сильное положение, согласно которому время само в какой-то мере определяет фундаменталь- 
ные характеристики материальных процессов» [2].

Действительно, понятию времени атомисты придают весьма примечательные функции. Однако, на наш взгляд, эти функции следовало придать не времени, а движению, поскольку, согласно функциональной концепции времени [3] не вечность времени обусловливает вечность движения, а наоборот, вечность движущейся материи обеспечивает вечность времени в том смысле, что в результате движения, изменений происходит возникновение и исчезновение объектов, образующих время. Иными словами, движение обусловливает время (в указанном смысле и вечность времени), а не время движение, вечность движения.

Вечность мира обусловливается не вечностью времени, а несотворимостью и неуничтожимостью материи, сохраняемой, а не изменяющейся стороной материи. Вечность мира - это вечность материи, неизменной стороны материи как субстанциональной реальности. Сама материя несменяема и неизменна при многообразии и сменяемости ее проявлений. Вечность мира связана с субстанцией, с материей, а вечность времени (в нашей интерпретации этого понятия) с субстанциональной сферой, т. е. с изменяющейся стороной субстанции.

Субстанциональная сфера находится в постоянном изменении, тогда как субстанция (сама материя) неизменна. С одной стороны, движение, изменения - причина становления, а потому и причина образования времени, с другой - движение, изменения обусловливают неизменность, сохраняемость, стабильность самой материи.

Таким образом, вечность мира - вечность того, что сохраняется, неизменно, стабильно, т.е. материи. А вечность времени - это вечность изменения, становления, возникновения и исчезновения, т.е. постоянного наличия во Вселенной конкретных форм материи, носящих преходящий характер, которые и образуют объективно-реальное, функциональное время, в котором и существуют.

Сказанное означает, что время присуще всем без исключения вещам, явлениям, процессам и их состояниям, но не материи, не миру в целом, поскольку никакие изменения не могут стать причиной исчезновения мира, материи, т. е. неизменной стороны субстанции [4].

Материя была материей в прошлом, будет материей и в будущем, потому что несотворима и неуничтожима.

Субстанциальную концепцию времени Ньютон ввел в физику. Она получила всеобщее признание в науке и сохранила ведущую роль вплоть до начала XX века. Вместе с тем концепция времени Ньютона явилась естественным продолжением и дальнейшим совершенствованием идей античных атомистов.
Как известно, А. Эйнштейн, отказался от классических представлений об абсолютном, ни от чего не зависящем времени. Тем самым, А. Эйнштейн вместо субстанциальной концепции времени И. Ньютона, предложил реляционную концепцию времени, в которой время уже не являлось физической сущностью.

Кроме того, А. Эйнштейн перешел к концепции, которая связывает время с реальными физическими процессами. Согласно указанной концепции «Пространственные и временные данные имеют не фиктивное, а физически реальное значение» [5].

Согласно учению Ньютона необходимо проводить различие между истинным, математическим или абсолютным временем и относительным, кажущимся или обыденным временем.

Так, Ньютон писал: «Абсолютное, истинное математическое время, само по себе и по самой своей сущности, без всякого отношения к чему-либо внешнему протекает равномерно и иначе называется длительностью.

Относительное, кажущееся или обыденное время есть или точная, или изменчивая, постигаемая чувствами, внешняя, совершаемая при посредстве какого-либо движения мера продолжительности, употребляемая в обыденной жизни вместо истинного математического времени, как-то: час, день, месяц, год» [6].

Вместе с тем объективно-реальное, функциональное время, образуемое конкретными материальными вещами, явлениями и процессами, в отличие от времени субстанциальной концепции, несубстанционально, хотя, тем не менее, объективно, поскольку с момента возникновения и до исчезновения материальных объектов, образующих его, имеет физическое значение. Сказанное не означает, что мы пытаемся умалить в какой-то степени значение концепции времени Ньютона, тем более, что сделать это невозможно, поскольку его постулаты об абсолютном времени (и пространстве) были совершенно необходимы для построения теоретической системы классической механики.

Помимо постулирования объективного характера времени (и пространства), концепция Ньютона имела еще неоспоримые методологические преимущества.

«До начала XX в., замечает Ю.Б. Молчанов, - субстанциальная концепция времени была единственной научной концепцией времени, из которой исходило естествознание прошлого века и особенно физика. Она продолжает играть позитивную и фундаментальную роль и в рамках современной науки, например классической механики и электродинамики, на законах которых основывается почти вся современная техника и 
производство. В настоящее время в наиболее глубоких теоретических областях современной физики, астрономии и космологии можно наблюдать действие в некотором смысле закона «отрицания отрицания», а именно возврат к субстанциальной трактовке времени на более высоком и сложном уровне» [7].

У Ньютона абсолютное время, как и абсолютное пространство, выступало ареной динамики физических объектов, тогда как объективно-реальное, функциональное время образуется благодаря этим изменениям, точнее в результате последовательной смены состояний конкретных физических объектов.

Во-первых, как полагал Ньютон «не существует (в природе) такого равномерного движения, которым время могло измеряться с совершенной точностью. Все движения могут ускоряться или замедляться, течение же абсолютного времени изменяться не может. Длительность или продолжительность существования вещей одна и та же, быстры ли движения, по которым измеряется время, медленны или их совсем нет» [6].

Во-вторых, и это главное: как уже подчеркивалось выше, объективное, функциональное время можно было бы измерить лишь «идеальными часами», т.е. процессом, который бы в точности мог повторять ритмы и длительности, образующиеся при последовательной смене состояний физического объекта, чье функциональное время требуется измерить.

Тем не менее функциональное время можно измерить обычными часами, но с учетом ритма и длительностей, образующиеся при последовательной смене состояний физического объекта.

Не является адекватным отражением объективнореального времени и относительное время концепции Ньютона. Дело в том, что, во-первых, как и другие концептуальные времена, оно также является постулированным, придуманным человеком временем, тогда как объективно-реальное время, как не раз подчеркивалось, это время, образуемое благодаря последовательной смене состояний конкретных материальных объектов.

По Ньютону, в отличие от объективно-реального времени, которое является отражением процесса, происходящим в данном объекте, как в едином целом, так и каждого из последовательно сменяющихся его состояния - «относительное пространство и время постигаемы чувствами» [6].

Не может отражать адекватно объективно-реальное относительное время и в том случае, если «относительное пространство и время... определяются системой отношений между материальными телами и событиями, поскольку функциональное время определяется, обусловливается последовательной сменой состояний конкретных материальных объектов и их самих.

В связи с вышесказанным не являются единицами объективно-реального времени такие единицы относительного, обыденного времени концепции Ньютона, как час, день, месяц, год, так же как и секунда или минута.

Тем не менее для большей ясности вспомним, что час состоит из определенного числа секунд и минут, а секунда, например, была определена как 1/86400 часть истинных солнечных суток, т. е. промежутка времени между двумя верхними положениями (кульминациями) центра видимости диска Солнца. Как известно, год определяется в связи с реальным движением Земли вокруг Солнца. Иными словами, определение единиц времени концепции Ньютона связывают с механическим движением с простым перемещением, тогда как объективно-реальное время образуется в результате последовательной смены состояний конкретных материальных объектов, образующих собственные временные длительности, которые так же последовательно сменяются.

Не является единицей объективно-реального времени даже секунда, определенная Международным комитетом по вопросам мер и весов в 1964 г. новым физическим эталоном времени как промежуток времени, в течение которого происходит 9192631770 колебаний электромагнитной волны, испускаемой атомом цезия-133, в отсутствие внешних электромагнитных полей, при переходе его из одного состояния в другое между двумя сверхтонкими уровнями энергии.

Объективно-реальным, функциональным временем является время самого атома цезия-133, образуемое последовательно сменяющимися промежутками времени, соответствующими последовательно сменяемым состояниям данного атома цезия. Однако, поскольку в природе не существует идеальных часов, которые бы при измерении, например, функционального времени атома цезия могли бы точно повторять и фиксировать ритмы и длительности, образуемые в результате последовательной смены состояний данного атома цезия, приходится пользоваться часами, которые измеряют длительность последовательно сменяющихся состояний, иными словами, промежутки времени, образуемые ими, т.е., единицы функционального времени. Из сказанного можно сделать вывод, что субстанциальная концепция времени будет играть позитивную роль не только в рамках современной науки, но и науки будущего.

Таким образом, определенные преимущества, которые имеет субстанциальная концепция, не связаны с адекватным отражением сущности объективно-реального времени или каких-то его свойств, а с тем, что их по- 
прежнему используют как в теории, так и на практике.

Что касается слабых сторон субстанциальной концепции времени, они, по справедливому мнению Ю.Б. Молчанова, «состоят в том, что постулируемое ею и необходимое для теоретической физики абсолютное время устанавливается только мысленно, т.е. в нашей голове, и ему, видимо, ничто не соответствовало в реальном мире... субстанциальная концепция может только постулировать, провозгласить установление временного порядка между событиями, ибо с ее точки зрения, временные отношения не связываются ни с какими физическими процессами или взаимодействиями» [8].

Не случайно субстанциональной концепции времени справедливо противопоставляют реляционную концепцию, которая рассматривает время как свойство или атрибут материи, как систему отношений между физическими событиями и телами. Эта система не является первичной, ни от чего не зависящей. Напротив, она не име- ет самостоятельного существования, а есть следствие, результат движения и взаимодействия материальных систем и событий.

В этой связи, Ю.Б. Молчанов, в результате анализа истории возникновения и эволюции субстанциальной и реляционной концепций времени, их взаимоотношения и конфронтации в свете современного уровня развития пришел к выводу об их взаимной диалектической дополнительности и выразил надежду на то, что в будущем, возможно, удастся добиться разумного синтеза и охватить в единой концепции различные и противоречивые аспекты, характеристики и стороны многоликого времени [7].

К сказанному лишь еще добавим, что, по нашему мнению, решение задачи создания единой концепции времени, на основе синтеза различных концепций времени, едва ли возможно, без функциональной концепции времени.

\section{ЛИТЕРАТУРА}

1. Материалисты Древней Греции. М.: Государственное издательство политической литературы, 1955. С. 55.

2. Молчанов Ю.Б. Четыре концепции времени в философии и физике. М.: Наука, 1977. С. 12.

3. Лолаев Т.П. Функциональная концепция времени: Диссертация на ... докт. филос. наук - М., 1993. - 273; Природа времени или время в природе: Теоретическое и экспериментальное обоснование объективного существования времени». М., 2015 и др.

4. Лолаев Т.П. Время как функция материального объекта, процесса // От времени теории относительности к объективно-реальному, функциональному времени. М.: «Научное обозрение» 2019. С. 32-43.

5. Эйнштейн А. Собр. науч. трудов. Т. ІІ. 1966. С. 24.

6. Ньютон И. Математические начала натуральной философии // Крылов А.Н. Собр. трудов. М. — Л., Наука - Изд-во Академии наук СССР, 1936. Т. 7. С. 30.

7. Молчанов Ю.Б. Проблема времени в современной науке. М.: Наука. 1990.

8. Молчанов Ю.Б. Проблема времени и диалектика // Вопросы философии. 1975. № 8. С. 83. 